\title{
Círculo trigonométrico: uma interface com o usuário para favorecer 0 aprendizado
}

\author{
Murilo U. Patutti ${ }^{1}$, Osmar Mantovani ${ }^{2}$ \\ ${ }^{1}$ Pesquisador Independente - Av. Ns. Sr. Graças, 2435 - Jd. Ns. Sr. Graças - Itatiba - \\ SP \\ ${ }^{2}$ Pesquisador Independente - Rua Líbano, 60 - Vila Brasileira - Itatiba - SP \\ \{muriloulhano, osmarmantovani\}@gmail.com
}

\begin{abstract}
This paper describes the development of an application for tablets to support the teaching of trigonometry in Brazilian high school courses. The work mainly aims detail the creation of the application's human-computer interface and evaluates its usability so that it can be used by teachers and students. The interface was evaluated by an expert using the usability inspection method and was compared with the usability of a tool similar to the proposal.
\end{abstract}

Resumo. Este artigo descreve o desenvolvimento de uma aplicação para tablets para apoiar o ensino de trigonometria em cursos brasileiros de ensino médio. O trabalho tem como objetivo principal detalhar a criação de interface humano-computador da aplicação e avaliar a sua usabilidade para que ela possa ser utilizada por professores e alunos. A interface foi avaliada por um especialista utilizando o método de inspeção de usabilidade e comparada com a usabilidade de uma ferramenta análoga à proposta.

\section{Introdução}

A educação básica corresponde aos primeiros anos da educação escolar. No Brasil, a educação básica compreende, de acordo com o Ministério da Educação, à educação infantil, ao ensino fundamental e ao ensino médio. Sua duração pode ultrapassar 12 anos. Em cada uma dessas três etapas, a escola trabalha para favorecer o desenvolvimento, no aluno, de uma série de competências e habilidades fundamentais para a sua vida. $\mathrm{O}$ ensino médio, em particular, é o momento no qual o estudante tem contato com diversas possibilidades de escolha para, por exemplo, o curso superior que pode cursar e o mercado de trabalho no qual pode atuar. Além disso, no contexto brasileiro, essa fase é marcada por uma grande preocupação com as provas de seleção para a universidade.

Nos últimos anos, novas tecnologias foram acrescentadas à sala de aula de ensino médio: computador, projetor, lousas digitais e equipamentos de som. O caso mais recente é o dos tablets. Escolas no mundo todo estão analisando essa tecnologia e a viabilidade da sua aplicação em sala de aula, de acordo com Schooltablets (2013). O governo federal brasileiro também possui iniciativas nesse sentido, como em MEC (2013). O uso de tablets no contexto educacional trás para o ambiente escolar o paradigma m-Learning descrito por Nyiri (2002), no qual, a oferta educativa ocorre, basicamente, através de dispositivos portáteis, de acordo com Traxler (2005). Segundo 
Shanmugapriya e Tamilarasi (2012), o m-Learning é o e-Learning através de dispositivos móveis e está ganhando força no mundo todo pela natureza da computação ubíqua e poderosa desses dispositivos. A utilização de dispositivos móveis no ambiente escolar pode trazer reflexos positivos para os alunos. Segundo Valente et al. (2009), estudo conduzido por Crawford e Vahey (2002) concluiu, através de vários indicadores, que tais dispositivos podem oferecer benefícios únicos aos alunos. Nas aulas de matemática do ensino médio brasileiro, no entanto, esta utilização atualmente é bastante reduzida. Além do atual número limitado de escolas brasileiras que provê acesso a essa tecnologia a seus professores e alunos, outra causa é a pequena quantidade de aplicativos disponíveis para tal tarefa. Uma busca rápida na loja online para aplicativos Android ${ }^{1}$ Google Play ${ }^{2}$ com as palavras chave Matemática Ensino Médio retorna 48 resultados que, em sua maioria, não são de aplicativos para ensino médio, e sim para ensino fundamental.

O tópico com currículo mais extenso dentro da Matemática para o ensino médio brasileiro é a Trigonometria. Em geral esse curso tem duração de cinco meses (dos nove meses de aula regular) e é apresentado aos alunos no segundo ano do ensino médio. Para uma contextualização simples da envergadura desse curso, das 470 páginas do livro de Iezzi et al. (2010), 300 abordam temas relacionados com Geometria. Dessas, 135 abordam Trigonometria ( $45 \%$ da parte do livro que trata de Geometria) sendo que, destas, cerca de $80 \%$ são baseadas no estudo e entendimento da trigonometria no chamado círculo trigonométrico, observado na Figura 1.

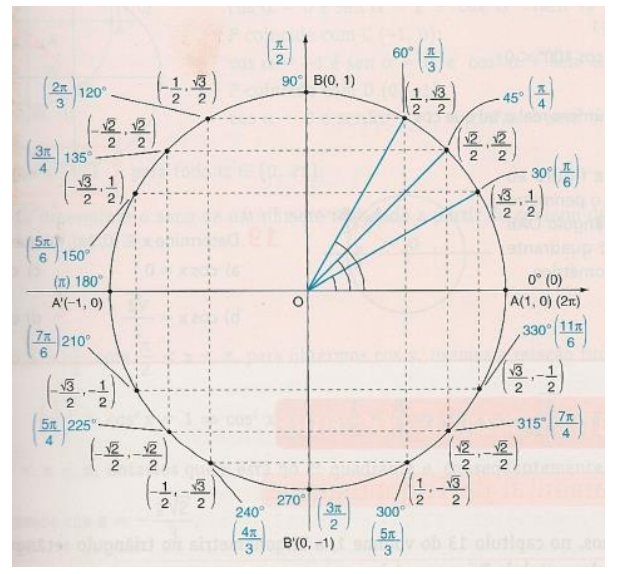

Figura 1. Círculo trigonométrico com arcos notáveis de lezzi et al. (2010).

A característica principal do círculo trigonométrico é que ele possui raio unitário. Observa-se na mesma figura que existem três exemplos de arcos indicados a partir do ponto $\mathrm{O}$, localizado no cruzamento dos eixos perpendiculares, também chamado de origem dos eixos. Considerando o arco de $30^{\circ}$ (ou $\frac{\pi}{6}$ radianos), por exemplo, sua extremidade localizada no ciclo, projetada perpendicularmente no eixo vertical, indica $\frac{1}{2}$. Este é o valor do seno de $30^{\circ}$.

\footnotetext{
${ }^{1}$ www.android.com, julho de 2013.

2 play.google.com, julho de 2013.
} 


\subsection{Problemas e Objetivo}

Uma palavra que ajuda a resumir o curso de Trigonometria é "projeção". As razões trigonométricas (seno, cosseno, tangente, cotangente, secante e cossecante) estão relacionadas com a projeção da extremidade do arco desejado (referenciado em graus ou em radianos) em locais específicos do círculo ou em retas a ele tangentes. Para explicar essa matéria, os professores, durante toda a parte do curso que envolve o círculo trigonométrico, recorrem, literalmente, a dezenas de desenhos na lousa. Dos livros didáticos também é exigido um esforço grande: Dante (2009), por exemplo, reproduz 59 vezes o ciclo no capítulo de trigonometria. A necessidade de reprodução na lousa custa ao professor um tempo muito grande e não permite interação para observar o que realmente acontece com as projeções durante a mudança do arco. Uma ferramenta computacional à qual aluno e professor tenham acesso e que permita a manipulação dos arcos e a consequente visualização de suas projeções pode favorecer a resolução destes problemas pedagógicos.

Uma busca no Google Play sobre "círculo trigonométrico" apresenta seis referências de aplicativos: dois envolvendo Séries de Fourier (abordagem avançada demais para alunos de ensino médio), dois envolvendo estudos em triângulos (não estando diretamente relacionados com o círculo trigonométrico) e outras duas fazendo referências a conteúdos estáticos (sem possibilidade de manipulação pelo aluno). Outras buscas no mesmo ambiente usando termos como "ciclo unitário" retornam poucas ou nenhuma referência. Nenhum dos aplicativos apresentados nestas respostas, no entanto, permite a manipulação de arcos no ciclo trigonométrico.

A única ferramenta disponível para plataforma Android que aborda o tema considerando as projeções da extremidade do arco, é a Trigonometric, de Pritron (2012), apresentada na Figura 2 e encontrada em buscas utilizando palavras da língua inglesa. Ela, no entanto, não possui versão em português, o que não favorece sua utilização em escolas brasileiras.

Outras características observadas na ferramenta em uma análise rápida da Figura 2 são: (a) valores precisos das razões trigonométricas; (b) as cores utilizadas nos segmentos do desenho são as mesmas utilizadas nas descrições e nos valores logo abaixo do círculo, favorecendo a identificação de oposto, adjacente, hipotenusa; (c) segmentos relacionados como hipotenusa e nova hipotenusa possuem a mesma cor, o que não permite sua identificação; (d) a definição das razões trigonométricas (cateto oposto por hipotenusa, por exemplo) é sempre apresentada, ocupando espaço da interface com o usuário e sem possibilidade de ser retirada; (e) Arcos notáveis são apresentados, mas de maneira que sua associação com a imagem não é simples (como o $217^{\circ}$ na Figura 2 (esquerda)); (f) Os pares ordenados indicados na Figura 2 (esquerda) estão sem parênteses, o que pode causar confusão, por exemplo, entre o par ordenado $(-1,0)$ e o número decimal -1,0 (um vírgula zero negativo ou simplesmente "menos um") (g) $\mathrm{Na}$ Figura 2 (direita), a abertura do arco, próxima à origem dos eixos, está indicada em vermelho, mas o valor do arco, identificado à esquerda, usa cor branca $\left(\right.$ Angle $=43^{\circ}$ ). Assim, numa análise rápida, conclui-se que a abordagem do software para o ensino é interessante, mas detalhes de usabilidade não favorecem algumas interpretações de conceitos matemáticos. 


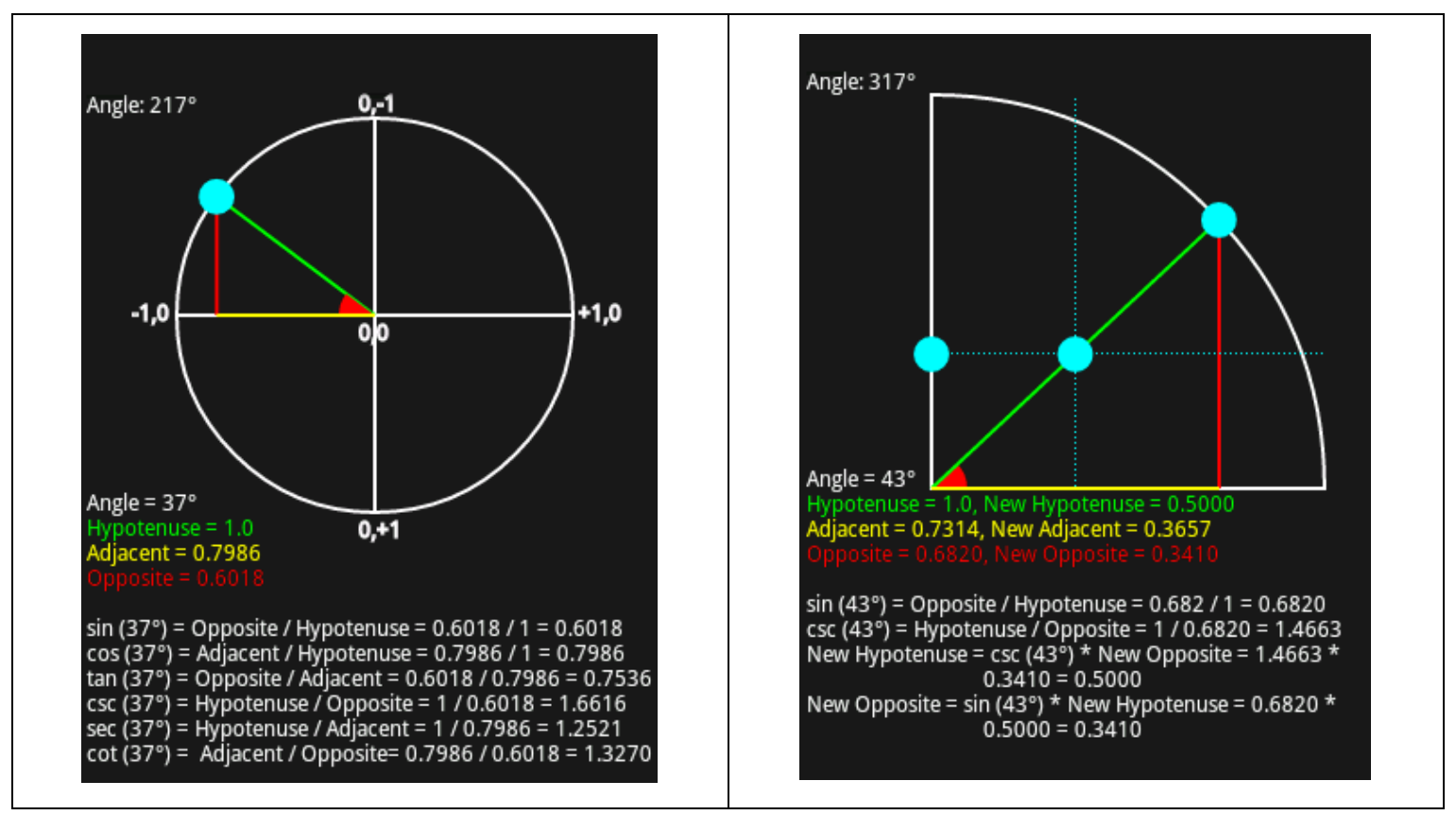

Figura 2. Ciclo trigonométrico (esquerda) e primeiro quadrante (direita) de Pritron (2012)

O objetivo deste trabalho é descrever o desenvolvimento e a avaliação da interface com o usuário de uma ferramenta computacional para tablets Android, a ser utilizada no ensino de trigonometria. Denominada "Círculo Trigonométrico", ela é parte de um sistema denominado ATPEM - Apoio Tecnológico para o Ensino Médio, e é resultante do trabalho de conclusão de curso de Murilo Ulhano Patutti em Engenharia de Computação pela Pontifícia Universidade Católica de Campinas no ano de 2012, sob coorientação do Prof. Ms. Osmar Mantovani. ATPEM é projetado para ser um arcabouço para outras ferramentas educacionais. "Círculo Trigonométrico" é a primeira delas.

Na seção 2 deste trabalho é feita uma descrição de referências bibliográficas relacionadas com aplicativos educacionais para tablets. Na seção 3 é descrito o processo de desenvolvimento da interface com o usuário do aplicativo "Círculo Trigonométrico". $\mathrm{Na}$ seção 4 são apresentados os resultados da análise da usabilidade do aplicativo proposto e, em seguida, as conclusões do trabalho.

\section{Referências}

Esta seção apresenta referências bibliográficas de aplicativos para tablets e celulares relacionadas com educação, com foco nas que oferecem suporte a algum tipo de interatividade ou comunicação professor-aluno.

O OrganicPad (Pargas et al., 2012) é uma ferramenta que utiliza tablets em sala de aula para o curso de química orgânica. Professor e alunos interagem durante a aula com a ferramenta, cada um em seu tablet, para resolver exercícios e entender as estruturas das moléculas orgânicas. As estruturas podem ser desenhadas pelos alunos ou somente visualizadas. A ferramenta permite ao professor criar exercícios e compartilhalos com os alunos durante as aulas ou fora delas, pois necessita da conexão com a Internet a todo o momento. 
O Mobile Math (Mahamad, 2012) visa complementar o ensino de matemática da educação básica na Malásia, através do m-learning utilizando telefones inteligentes e PDAs (Personal Digital Assistant). Como é uma complementação ao ensino, ele é utilizado basicamente para os professores fornecerem desafios aos alunos interessados. Esses desafios podem ser descritos como simples perguntas matemáticas ou resultados de operações básicas como a soma, divisão, subtração e multiplicação.

O sistema Mobile Learning Environment System (MLES) (Hanafi e Samsudin, 2012) é um ambiente para o ensino que fornece conteúdo educativo e promove interatividade entre alunos e professores, pois está presente em diversas plataformas, celulares e tablets Android e computadores de mesa. Através dele, alunos e professores resolvem exercícios, mandam mensagens uns para os outros para tirar dúvidas, compartilham conteúdos relacionados com alguma matéria e obtém material de aula. $\mathrm{O}$ MLES ainda fornece aos professores a opção de criar pesquisas, informativos e também fornecer informações instantaneamente a seus alunos.

Como se pode observar nenhum dos sistemas analisados apresenta preocupação explícita com o desenvolvimento e/ou análise da interface com o usuário.

\section{A interface com o usuário}

Uma das preocupações de Constantine e Lockwood (1999) é o que o usuário vai fazer na maioria do tempo quando ele está utilizando o sistema. Segundo a proposta de Liesenberg e Mantovani (2005) o Círculo Trigonométrico possui dois principais perfis de usuários: Professores e Alunos; e eles estarão, na maior parte do tempo, observando projeções e valores das razões trigonométricas no círculo. Considerando isto e também que o outro aplicativo do gênero (Trigonometric) citado na seção 1.1, e que poderia ser utilizado para comparação na avaliação, apresenta apenas o círculo trigonométrico para ser explorado, o esforço no desenvolvimento da interface com o usuário levou em conta o círculo trigonométrico como elemento funcional fundamental.

Assim, surgiram outros elementos funcionais também colocados no primeiro protótipo da interface: botões relacionados com os arcos notáveis e com as razões trigonométricas e dois botões específicos para facilitar a localização de arcos (ajuste fino, visto que o ajuste grosso poderia ser feito deslizando-se a extremidade do arco no círculo). Cada protótipo foi analisado por um especialista em usabilidade. Sua análise foi feita com base em aspectos técnicos a serem descritos e em tarefas típicas, como proposto por Constantine e Lockwood (1999). Os resultados de cada análise foram utilizados para implementar melhorias na versão seguinte da interface.

\subsection{Primeiro Protótipo da Interface}

Apesar de ter sido criada sem a utilização de ferramentas computacionais, o protótipo era em uma folha de sulfite do tamanho exato da tela de sete polegadas do tablet Samsung modelo GT-P6210 com a versão 3.2 (Honeycomb) do Android, escolhido para o desenvolvimento. Segundo Liesenberg (2012), “a construção de protótipos em papel é uma técnica clássica de grande aceitação no meio dos especialistas em desenvolvimento de interfaces de usuário devido à sua simplicidade, ao seu baixo custo e por ser muito efetiva". 
Além da tela, o protótipo, cuja representação é apresentada na Figura 3 era composto de dois post-its: (a) um deles com o ciclo trigonométrico desenhado e, nele, um arco de $20^{\circ}$ e suas projeções e (b) outro com o retângulo com os dados de seno, cosseno e tangente de $20^{\circ}$. Tais materiais foram apresentados ao analista de usabilidade que, neste momento, assumiu o papel de um professor de matemática tentando realizar uma tarefa típica: determinar o valor do seno do arco de $20^{\circ}$. Ao manipular os post-its, quando a intenção do usuário foi a de deslocar extremidade do arco até 20 graus, eles foram colocados sobre o ciclo trigonométrico original indicando o arco selecionado (e suas projeções) e sobre o retângulo com os valores das razões trigonométricas. O usuário pôde então observar o valor do seno procurado, concluindo a tarefa típica.

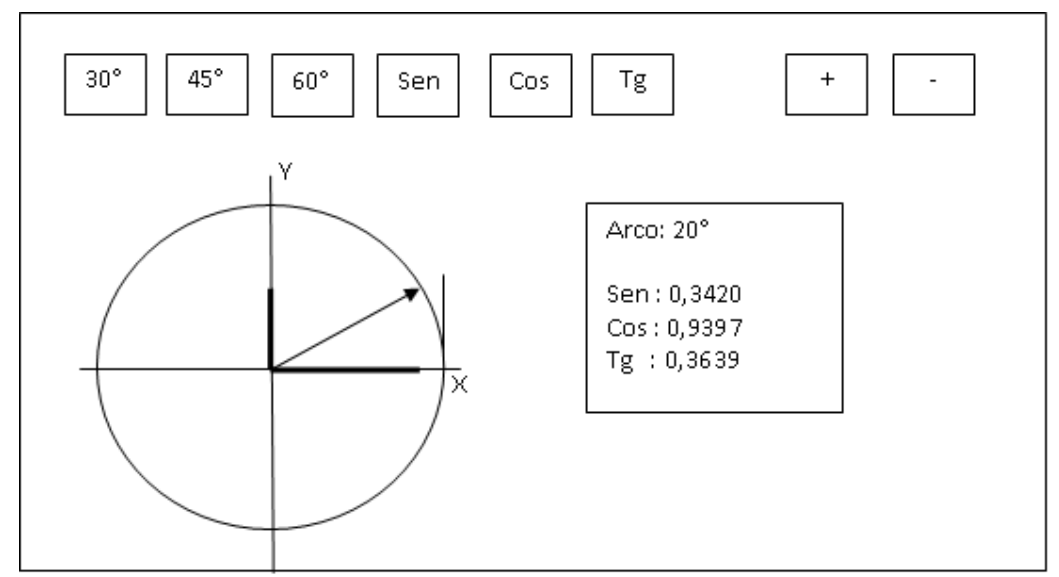

Figura 3: Representação do primeiro protótipo da interface, originalmente feito à mão e em folha de sulfite.

As principais observações resultantes da manipulação do protótipo e dos demais materiais a ele associados, foram utilizadas para a elaboração do segundo protótipo em papel.

\subsection{Segundo Protótipo da Interface}

As principais alterações advindas das observações anteriores foram: (a) o círculo dividido a cada 10 graus; (b) o deslocamento dos botões +/- para a direita; (c) o reposicionamento dos botões das razões trigonométricas e dos arcos notáveis e (d) a apresentação dos valores das razões trigonométricas na parte superior da tela.

O especialista em usabilidade foi novamente convidado a experimentar este novo protótipo em papel e, desta vez, não teve nenhuma dificuldade em realizar a tarefa típica anteriormente descrita. Foi, então, convidado a realizar outra tarefa típica: observar que o seno e o cosseno de 45 graus têm o mesmo valor. As principais observações que surgiram a partir da realização desta tarefa, novamente, utilizadas para a elaboração do terceiro protótipo em papel.

\subsection{Terceiro Protótipo e Versão Final da Interface}

A partir destas observações, uma nova versão, a última em papel, foi elaborada. Uma decisão importante tomada foi que as indicações das divisões do ciclo a cada $10^{\circ}$ deveriam ser mais discretas. 
A partir deste terceiro protótipo foi criada então a primeira versão da interface do Círculo Trigonométrico, único plug-in do sistema ATPEM até o momento. Posteriormente a avaliação da usabilidade da mesma foi realizada pelo especialista. É importante ressaltar que a escolha da prototipação em papel para iniciar $o$ desenvolvimento da interface com o usuário contribuiu fortemente para que a versão final da tela, Figura 4, fosse obtida rapidamente. Assim, a avaliação da usabilidade a ser apresentada a seguir foi favorecida pelas inspeções de usabilidade, realizadas quando da prototipação em papel.

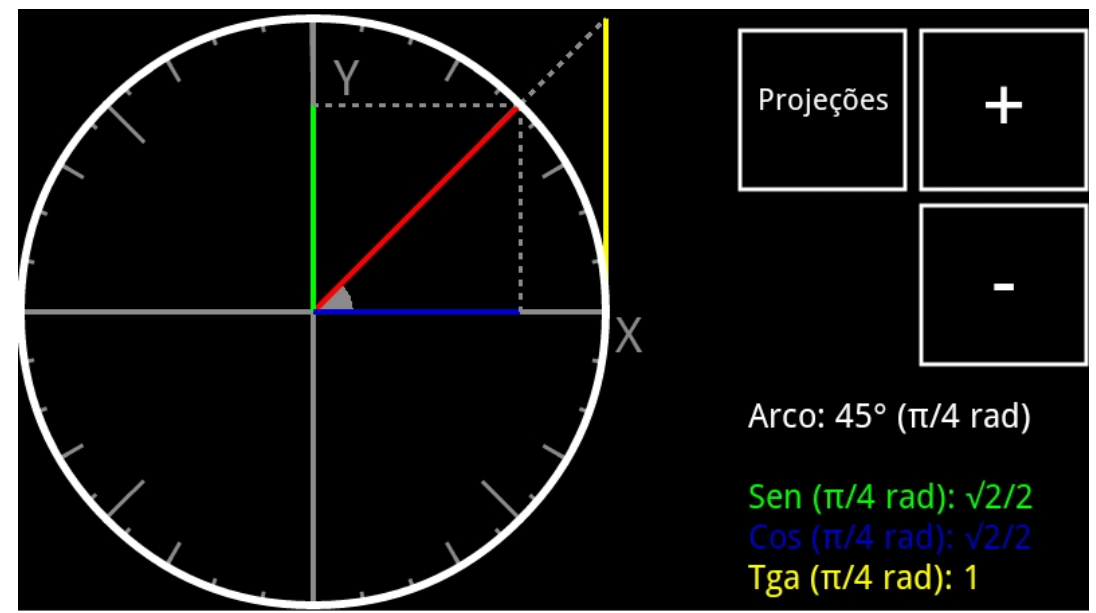

Figura 4. Versão final da interface com o usuário do aplicativo "Ciclo Trigonométrico", plug-in do ATPEM.

\section{Usabilidade}

Nesta seção é descrita a avaliação da usabilidade do aplicativo Círculo Trigonométrico. Ela foi feita por um especialista com base em padrões de usabilidade reconhecidos e também comparando-se com a usabilidade do sistema Trigonometric, cuja funcionalidade principal está diretamente relacionada com a deste trabalho: a exploração de arcos no círculo trigonométrico e suas projeções.

\subsection{Avaliação da Usabilidade}

Para a avaliação da usabilidade da interface com o usuário o especialista utilizou o método de inspeção de usabilidade. Segundo Rocha e Baranauskas (2000), "Inspeção de usabilidade objetiva encontrar problemas de usabilidade em um design de uma interface de usuário e, com base nesses problemas, fazer recomendações no sentido de eliminar os problemas e melhorar a usabilidade do design. Isso significa que inspeções de usabilidade são feitas em um estágio onde a interface está sendo gerada e a sua usabilidade (e utilidade) necessita ser avaliada". 


\section{Quadro 1 - Questionário de avaliação de usabilidade - Aplicativo Círculo} Trigonomético

\begin{tabular}{|c|c|c|c|c|c|c|c|c|}
\hline Questões & & 1 & 2 & 3 & 4 & 5 & & $\begin{array}{l}\text { Sem } \\
\text { resposta }\end{array}$ \\
\hline \multicolumn{9}{|l|}{ Satisfação } \\
\hline Começar foi... & Difícil & & & & & $\mathrm{X}$ & Fácil & \\
\hline $\begin{array}{l}\text { Quanto a forma, tamanho e realces } \\
\text { (ícones, cores, letras, negrito) nas telas: }\end{array}$ & $\begin{array}{l}\text { Pouco } \\
\text { legível }\end{array}$ & & & & & $\mathrm{X}$ & Muito legível & \\
\hline $\begin{array}{l}\text { Apresenta uma distribuição uniforme do } \\
\text { conteúdo, levando em consideração o espaço } \\
\text { disponível? }\end{array}$ & Sempre & & & & $\mathrm{X}$ & & Nunca & \\
\hline $\begin{array}{l}\text { Informa o usuário sobre o que está } \\
\text { acontecendo? (por exemplo: que a execução } \\
\text { está em andamento) }\end{array}$ & Nunca & & & & & $\mathrm{X}$ & Sempre & \\
\hline \multicolumn{9}{|l|}{ Aprendizado } \\
\hline $\begin{array}{l}\text { O tempo para aprender a utilizar o aplicativo } \\
\text { e seus recursos foi... }\end{array}$ & $\begin{array}{l}\text { Pouco } \\
\text { tempo }\end{array}$ & $\mathrm{X}$ & & & & & Muito tempo & \\
\hline $\begin{array}{l}\text { Os ícones e/ou botões utilizados nas telas são } \\
\text { familiares, isto é, lembram o que se deve } \\
\text { fazer? }\end{array}$ & Nunca & & & & & $\mathrm{X}$ & Sempre & \\
\hline $\begin{array}{l}\text { Se você ficasse algum tempo sem utilizar o } \\
\text { aplicativo, na próxima vez que fosse usar, ele } \\
\text { seria... }\end{array}$ & Dificil & & & & & $\mathrm{X}$ & Fácil & \\
\hline $\begin{array}{l}\text { As tarefas podem ser executadas de uma } \\
\text { maneira rápida e/ou lógica? }\end{array}$ & Nunca & & & & & $\mathrm{X}$ & Sempre & \\
\hline \multicolumn{9}{|l|}{ Erros } \\
\hline $\begin{array}{l}\text { O aplicativo apresenta telas de mensagens de } \\
\text { erros, quando um erro ocorre? }\end{array}$ & Nunca & & & & & & Sempre & $X$ \\
\hline $\begin{array}{l}\text { As mensagens de erro ajudam a solucionar o } \\
\text { problema? }\end{array}$ & Nunca & & & & & & Sempre & $\mathrm{X}$ \\
\hline $\begin{array}{llll}\text { As mensagens de erro } & \text { estão } & \text { bem } \\
\text { posicionadas ou legíveis? } & & \\
\end{array}$ & Nunca & & & & & & Sempre & $\mathrm{X}$ \\
\hline Quanto ao texto, as mensagens de erro são... & Confusas & & & & & & Muito Claras & $\mathrm{X}$ \\
\hline \multicolumn{9}{|l|}{ Memorização e Eficiência } \\
\hline $\begin{array}{l}\text { A velocidade de resposta durante a realização } \\
\text { das tarefas foi... }\end{array}$ & $\begin{array}{l}\text { Muito } \\
\text { lenta }\end{array}$ & & & & & $X$ & Aceitável & \\
\hline $\begin{array}{l}\text { Seu uso depende do nível de experiência do } \\
\text { usuário? }\end{array}$ & Nunca & & & $\mathrm{X}$ & & & Sempre & \\
\hline $\begin{array}{l}\mathrm{O} \text { usuário realiza as tarefas com pouco } \\
\text { conhecimento? }\end{array}$ & $\begin{array}{c}\text { Com } \\
\text { dificuldade }\end{array}$ & & & $\mathrm{X}$ & & & Facilmente & \\
\hline $\begin{array}{l}\text { Depois de um ou dois dias sem utilizar o } \\
\text { aplicativo, quando voltou a usar foi... }\end{array}$ & Dificil & & & & & $\mathrm{X}$ & Fácil & \\
\hline As informações apresentadas na "tela" são... & $\begin{array}{l}\text { Desneces- } \\
\text { sárias }\end{array}$ & & & & $\mathrm{X}$ & & Necessárias & \\
\hline
\end{tabular}

As inspeções de usabilidade foram aplicadas através do método de avaliação heurística, descrito por Nielsen (2012). Como colocado anteriormente, a avaliação da interface com o usuário não se deu apenas com a versão final da interface concluída e sendo executada no tablet, ela foi realizada com a versão extremamente próxima da final, sendo executada no tablet, mas, antes disso, durante a prototipação em papel. $\mathrm{O}$ processo realizado desta maneira deu mais segurança ao desenvolvedor que pôde compreender melhor como é o processo de criação de uma interface com o usuário com boa usabilidade. 
A interface do Trigonometric estava, evidentemente, pronta. Logo, a avaliação da sua interface não é muito favorecida (o ideal seria fazer a avaliação de versões intermediárias também), mas é interessante para que se possa realizar uma comparação com a que foi criada para o "Círculo Trigonométrico", primeiro plug-in do ATPEM.

Um momento importante, evidentemente, foi o da avaliação final da usabilidade pelo especialista. O desenvolvedor teve a oportunidade de vivenciar esse momento com certa tranquilidade, pois a interface proposta não estava sendo apresentada pela primeira vez. Assim, a avaliação final da usabilidade foi muito boa, como pode ser observado no questionário, apresentado no Quadro 1.

\section{Conclusões}

Para efetivamente verificar se o objetivo de conseguir melhor usabilidade foi atingido, uma comparação entre as médias das notas por item do questionário foi realizada. Sendo que maior a nota, melhor o resultado. A comparação pode ser observada no Quadro 2. Como as notas do Círculo Trigonométrico são maiores que as do Trigonometric, conclui-se que o objetivo do trabalho foi alcançado.

Quadro 2. Comparativo de notas médias

\begin{tabular}{|l|c|c|}
\hline \multicolumn{1}{|c|}{ Características } & Trigonometric & $\begin{array}{c}\text { Círculo } \\
\text { Trigonométrico }\end{array}$ \\
\hline Satisfação & 4,25 & 4,75 \\
\hline Aprendizado & 3,00 & 4,00 \\
\hline Memorização e eficiência & 3,80 & 4,00 \\
\hline Erros & N/A & N/A \\
\hline
\end{tabular}

Este trabalho apresentou o desenvolvimento da interface com o usuário do Círculo Trigonométrico, aplicativo para tablets Android e primeiro plug-in do sistema ATPEM - Apoio Tecnológico Para o Ensino Médio, que, pretendemos, seja um arcabouço tecnológico para outras ferramentas voltadas à educação. As principais contribuições do desenvolvimento da interface com o usuário deste aplicativo são: (a) tecnológica, ao iniciar um padrão, baseado em critérios científicos, que pode direcionar a criação das interfaces com o usuário de futuros plug-ins do sistema e (b) pedagógica, ao criar uma ferramenta educacional simples e eficaz com uma interface que favoreça sua utilização.

O aplicativo está disponível, gratuitamente, na Google Play, em fase de testes.

\section{Referências}

Crawford, V.; Vahey, P. Palm Education Pioneers Program: March, 2002 Evaluation Report, In SRI International, Estados Unidos.

Constantine, L.; Lockwood, L; Software for Use, ACM Press, New York, $1^{\text {a }}$ Ed., 1999.

Dante, L. R. Matemática contexto e aplicações. 3. ed. Volume único. São Paulo, Brasil: Ática, 2009. 
Hanafi, F.H.; Samsudin, K. Mobile Learning Environment System (MLES): The Case of Android-based Learning Application on Undergraduates' Learning. International Journal of Advanced Computer Science and Applications. Vol.3, No.3, 2012.

Iezzi, G.; Dolce, O.; Degenszajn, D.; Périgo, R.; Almeida, N.; Matemática, Ciência e

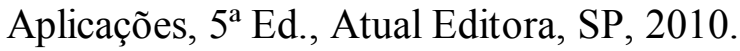

Liesenberg, H.; Interfaces de usuário. https://sites.google.com/a/liesenberg.biz/interfaces, Outubro de 2012.

Liesenberg, H.; Mantovani, O.; YAI: Atores de um sistema de compartilhamento de conteúdos para uma educação solidária; XI Workshop sobre Informática e na Escola (WIE); Unisinos; São Leopoldo; 2005.

Mahamad, S. Open Source Implementation of M-Learning for Primary School in Malaysia. International Journal of Human and Social Sciences. Malásia, 2008.

MEC, "MEC vai distribuir 600 mil tablets a professores do ensino médio", http://redecomunicadores.mec.gov.br/index.php?option=com_content\&view $=$ article \& $\mathrm{id}=3810$ :mec-vai-distribuir-600-mil-tablets-a-professores-do-ensinomedio\&catid $=93$ : noticiasrede\&Itemid $=232$, Julho de 2013.

Nielsen, Definition of usability. Nielsen Norman Group, http://www.useit.com/alertbox/20030825.html, Abril de 2012.

Nielsen, Ten Usability Heuristics. Nielsen Norman Group, http://www.useit.com/papers/heuristic/heuristic_list.html. Março de 2012.

Nyiri, K. Towards a philosophy of m-Learning. International Workshop on Wireless and Mobile Technologies in Education. Washington, Estados Unidos. 2002.

Pargas, R.; Cooper, M.; Willians, C.; Bryfczynski, S. OrganicPad: A Tablet PC Based Interactivity Tool for Organic Chemistry. First International Workshop on Pen-Based Learning Technologies. Clemson, Carolina do Sul, Estados Unidos, 2007.

Pritron, “Trigonometric", <https://play.google.com/store/apps/details?id=net.pritron. android.trigonometric\&feature=search_result $>$. Março de 2012.

Rocha, H.V.; Baranauskas, M.C.C. Design e avaliação de interfaces humanocomputador. Campinas: CopySet, 2000.

Schooltablets, "International Schools With Tablet Computer Initiatives", http://www.schooltablets.org. Julho de 2013.

Shanmugapriya, M.; Tamilarasi, A. Developing a Mobile Adaptive Test (MAT) in an MLearning Environment for Android Based 3G Mobile Devices. International Journal on Computer Science and Engineering (IJCSE). Estados Unidos, 2012.

Traxler, J., Defining mobile learning. IADIS International Conference Mobile Learning. Berlin, Alemanha, 2005.

Valente, J.A.; Marçal, E.; Ribeiro, J.W.; Lima, L.; júnior, M.; Andrade, R. XV Workshop Sobre Educação na Escola. Bento Gonçalves, RS, 2009. 